\title{
A New Fluorescent Method to Detect Sulfamidase Activity in Blood, Tissue Extracts and Dried Blood Spots
}

Journal of Inborn Errors of Metabolism \& Screening 2021, Volume 9: e20200021 DOI: https://doi.org/10.1590/2326-4594JIEMS-2020-0021

\author{
Xingxuan He', Edward H. Schuchman' and Calogera M. Simonaro' ${ }^{1}$
}

\begin{abstract}
Mucopolysaccharidosis type IIIA (MPS IIIA) is a lysosomal storage disorder due to the deficient activity of sulfamidase (SGSH). Traditionally, measurement of this enzymatic activity has been performed using a fluorescently (4-MU) labeled glycoside substrate. While this substrate is inexpensive and readily available, the current method requires a 2-step procedure that is performed over 2 days. Here we report a new and simplified procedure using the 4-MU substrate. Major advantages of this assay method over the existing fluorescent method include a single step vs. 2-step procedure, an incubation time of 1 hour, and high sensitivity. The reaction is also run on UPLC equipment, which is available in most research labs and permits separation of the endogenous, autofluorescent material from the 4-MU signal. This assay method was developed using the MPS IIIA mouse model, and was validated using mouse plasma, liver and brain extracts, and dried blood spots. Human MPS IIIA skin fibroblasts and dried blood spots also were used to validate the method.
\end{abstract}

Keywords: Mucopolysaccharidosis type IIIA, Sanfilippo Syndrome, sulfamidase, diagnostic assay.

\section{Introduction}

Mucopolysaccharidosis type IIIA (MPS IIIA; Sanfilippo Syndrome type A) is a lysosomal storage disorder due to the inherited deficiency of sulfamidase (SGSH; E.C. 3.10.1.1) activity [1]. SGHS is one of several enzymes included in the breakdown of the glycosaminoglycan, heparan sulfate, resulting in its accumulation in most tissues [2]. Over time, the accumulation of heparan sulfate leads to lysosomal dysfunction, chronic inflammation, cell death and disease.

Due to the critical importance of heparan sulfate in brain development, MPS IIIA presents as a chronic and severe neurologic disorder [3]. Early signs of the disease include speech delay, poor sleep, developmental delay, "autistic-like" behavior, and hyperactivity. It is generally diagnosed within the first decade of life, and as patients age they often experience seizures, loss of mobility, dystonia, dementia and finally death. As with all lysosomal storage disorders, the degree of clinical involvement varies greatly among patients. Other organ systems also may be affected beyond the central nervous system, including the liver and skeletal systems. No disease-specific therapies are available for the disorder, although gene therapies and other approaches are under investigation $[4,5]$.

MPS IIIA is inherited as an autosomal recessive trait, and diagnosis of the disorder requires confirmation of the markedly reduced SGSH activity. Civallero et al. have used the traditional, method using a fluorogenic 4-Methylumbellieryl (4-MU) labeled glycoside substrate to measure SGSH activity in dried blood spots (DBS) from MPS IIIA patients [6]. The procedure requires a time consuming 2 -step procedure that is performed over 2 days, and the results are generally quantified using a fluorometer or 96-well plate reader [7]. The sensitivity of this equipment is generally low, and the inability to separate the endogenous, autofluorescence present in many biological samples from the 4-MU signal can lead to quenching and other artifacts. Therefore, despite the fact that the method uses an inexpensive, commercial substrate, very few laboratories have adapted it.

In addition to the fluorogenic method, mass spectrometry assay methods also have been developed. These methods have high sensitivity and specificity, and have been adapted to DBS [8-10]. However, they require the use of mass spectrometry

\footnotetext{
${ }^{1}$ Icahn School of Medicine at Mount Sinai, Department of Genetics \& Genomic Sciences, 1425 Madison Avenue, 10029, New York, NY, USA.
}

Received November 18, 2020, and in revised form January 09, 2021. Accepted for publication January 20, 2021.

\section{Corresponding Author:}

Calogera M. Simonaro, Icahn School of Medicine at Mount Sinai, Department of Genetics \& Genomic Sciences, 1425 Madison Avenue, 10029, New York, NY, USA.

Email: calogera.simonaro@mssm.edu 
equipment that may not be readily accessible in some research laboratories.

We have therefore developed an alternative method using the 4-MU substrate and UPLC equipment. This method offers considerable advantages over the existing 4-MU assay, including a 1-step vs. 2-step procedure, $1 \mathrm{hr}$ incubation time vs. 2 days, and markedly enhanced sensitivity. The method has been validated using MPS IIIA mouse plasma, liver and brain extracts, and DBS, as well as human MPS IIIA skin fibroblasts and DBS.

\section{Materials and Methods}

\section{Materials}

4-Methylu mbellieryl-2-sulfamino-2-deoxy-alph-Dglucopyranoside (4MU-GlcNS) was from Carbosyth (San Diego, CA, USA). Recombinant mouse SGSH was purchased from R\&D Systems (Minneapolis, MN, USA). Alpha-glucosidase $(\alpha \mathrm{GD})$ from Saccharomyces cerevisiae was from Sigma (St. Louis, MO, USA). DBS saver cards were from PerkinElmer (Greenville, SC, USA). A protein assay kit was purchased from Bio-Rad (Hercules, CA, USA). All other chemicals were from Thermo Scientific (Waltham, MA, USA).

\section{MPS IIIA Mouse Colony}

MPS IIIA and age-matched wild type and heterozygous mice [11] were bred, housed, and maintained in the animal facility of the Icahn School of Medicine at Mount Sinai. All animal protocols were approved by the Mount Sinai Institutional Animal Care and Use Committee (protocol \#08-0108), and were performed in accordance with NIH guidelines. All mice were genotyped using established protocols [12]. Euthanasia was performed using carbon dioxide inhalation.

\section{Mouse Plasma and Tissue Homogenate Preparation}

Mouse blood was collected into a heparin (green top) tube. The supernatant was transferred into a $1.5 \mathrm{ml}$ Eppendorf tube after centrifugation at 2,000 $\mathrm{g}$ for $10 \mathrm{~min}$ at $4^{\circ} \mathrm{C}$. The plasma was then used immediately for the SGSH enzyme activity assay or stored at $-20^{\circ} \mathrm{C}$. Mouse brain and liver were homogenized in $10 \%(\mathrm{w} / \mathrm{v})$ cell lysis buffer (20 mM HEPES buffer, pH 7.5, $150 \mathrm{mM} \mathrm{NaCl}$, $0.2 \%$ Igepal, $1 \times$ protease inhibitor cocktail) and centrifuged at $10,000 \mathrm{~g}$ for $10 \mathrm{~min}$ at $4^{\circ} \mathrm{C}$. The supernatant was then used for the enzyme activity assay or stored at $-20^{\circ} \mathrm{C}$ for future use.

\section{Dried Blood Spot Preparation}

To prepare mouse DBS, $20 \mu \mathrm{l}$ of mouse blood was spotted onto a Perkin Elmer Spot Saver Card. The cards were air dried under a hood for $4 \mathrm{hr}$, and then wrapped and stored in a desiccant chamber at $4^{\circ} \mathrm{C}$ for over 4 weeks. To validate the method for humans, a DBS card was received with blood from a known MPS IIIA patient and heterozygous parent. The samples were de-identified and informed consent was obtained. For our standard SGSH assay, a $3 \mathrm{~mm}$ (diameter) DBS was punched from the mouse or human cards and transferred to a $1.5 \mathrm{ml}$ tube containing $10 \mu \mathrm{l}$ of saline. After $1 \mathrm{hr}$ incubation with slow shaking at $37^{\circ} \mathrm{C}, 10 \mu \mathrm{l}$ of substrate buffer was added and incubated for an additional $1 \mathrm{hr}$. The reaction solution was then ready for the SGSH assay using the Acquity UPLC equipment (see below).

\section{Human MPS IIIA Skin Fibroblast Preparation}

Human skin fibroblasts from two MPS IIIA heterozygous donors (GM00886 and GM01094) and one homozygous patient (GM00879) were obtained from the Coriell Institute (Camden, NJ, USA) and cultured in DMEM containing $15 \%$ fetal bovine serum [13]. After reaching $~ 80 \%$ confluency, the cells were harvested by trypsinization, centrifuged at $1500 \mathrm{~g}$ for $10 \mathrm{~min}$, and the cell pellets collected. The pellets were washed twice in Hank's balanced salt solution and then frozen at $-20^{\circ} \mathrm{C}$. To determine SGSH activity in these cell pellets, a cell lysate was prepared by re-suspending them in lysis buffer $(20 \mathrm{mM}$ HEPES buffer, $\mathrm{pH} 7.5,150 \mathrm{mM} \mathrm{NaCl}, 0.2 \%$ Igepal, $1 \times$ protease inhibitor cocktail). After five cycles of freeze-thaw and centrifugation at $10,000 \mathrm{~g}$ for $10 \mathrm{~min}$ at $4^{\circ} \mathrm{C}$, the supernatants were ready for enzyme activity assays as described below.

\section{SGSH Activity Assay}

Our newly developed SGSH assay was carried out using a mixture of $5 \mu \mathrm{l}$ each of sample and substrate buffer $(0.8 \mathrm{mM} 4 \mathrm{MU}-\mathrm{GlcNS}$, 0.2 units/ $\mu \mathrm{l} \alpha \mathrm{GD}, 100 \mathrm{mM}$ HEPES buffer [pH 6.5], $200 \mathrm{mM}$ $\mathrm{CaCl}_{2}$ ). Ten $\mu \mathrm{l}$ of saline (for plasma) or lysis buffer (for liver or brain homogenates, or human skin fibroblast lysates) was used as a blank. For the DBS, a $3 \mathrm{~mm}$ punch from an empty PerkinElmer spot saver card was incubated with saline and then used as a blank. After incubation at $37^{\circ} \mathrm{C}$ for $1 \mathrm{hr}$, the $10 \mu \mathrm{l}$ reaction was stopped by addition of $50 \mu \mathrm{l}$ of sodium carbonate buffer $(\mathrm{pH}$ $10.8)$ and centrifuged (13,000 g for $10 \mathrm{~min})$. Supernatants were transferred to sample vials and analyzed using an Acquity $\mathrm{H}$-Class UPLC system equipped with a fluorescence detector (model B10UPF823M) and BEH Shield RP18 $1.7 \mathrm{~mm}$ 2.1x50 mm column (Waters, Milford, USA). The gradient system was $20 \mathrm{mM}$ ammonium hydroxide for mobile phase A and 100\% acetonitrile for mobile phase $\mathrm{B}$. The gradient program was: 0-0.3 min 98\%-50\% A, 2-50\% B; 0.3-0.4 min 50-98\% A, 50-2\% B; 0.4-1.4 $\mathrm{min} 98 \% \mathrm{~A}, 2 \% \mathrm{~B}$, at a flow rate of $1 \mathrm{ml} / \mathrm{min}$. The fluorescent product (4-MU) was monitored at an excitation wavelength of $365 \mathrm{~nm}$ and an emission wavelength of $460 \mathrm{~nm}$. Quantification of the 4-MU peak was performed using the Waters Empower Software according to a 4-MU standard curve. For some comparative experiments, the 4-MU signal from these reactions also was determined using a 96 -well fluorescent plate reader (BioTek Synergy HT). 


\section{Statistical Analysis}

For two group comparisons, the Mann-Whitney U test for nonparametric data or a two sample Student's t test for data with parametric distribution was used. For multiple comparisons, data with a normal distribution were analyzed by two ways ANOVA followed by Bonferroni or Games-Howell post hoc test. GraphPad Prism 5.0 software (GraphPad Software, La Jolla, CA, USA) was used for all statistical analysis.

\section{Results}

\section{Separation of Autofluorescent and 4-MU Signals by UPLC}

4MU-GlcNS has been commonly used as a substrate to determine SGSH activity [7]. The standard procedure requires two independent hydrolysis steps and is performed over two days. The final 4-MU product emits fluorescence that is generally measured using a 96-well plate reader or fluorometer. This method is time consuming and has low sensitivity. Here, we have developed a sensitive, rapid and reliable method to determine SGSH activity using the 4-MU substrate and a UPLC system.

Our first goal was to develop a UPLC method that could separate the 4-MU signal from the endogenous, autofluorescent material present in most biological samples. This is an important distinction of our method from the current 4-MU method since fluorometers or plate readers cannot separate these signals, which could potentially interfere with the results. As shown in Fig. 1 using mouse spleen extracts, which has some of the highest levels of autofluorescent material, this signal could be readily separated from the 4-MU peak using our UPLC system.

\section{Development of a One-Step Reaction and Condition Optimization}

As mentioned above, the current 4-MU SGSH assay requires two steps to measure activity: in the first step $4 \mathrm{MU}-\mathrm{GlcNS}$ is desulfated by SGSH present in the test biological sample to produce $4 \mathrm{MU}-\mathrm{Glc}$, a substrate for $\alpha \mathrm{GD}$, which further converts $4 \mathrm{MU}-\mathrm{Glc}$ to $4-\mathrm{MU}$ in the second step. The fluorescence of the 4-MU product is then quantified using either a plate reader or a fluorometer. Based on the buffer preferences of SGSH and $\alpha \mathrm{GD}$,

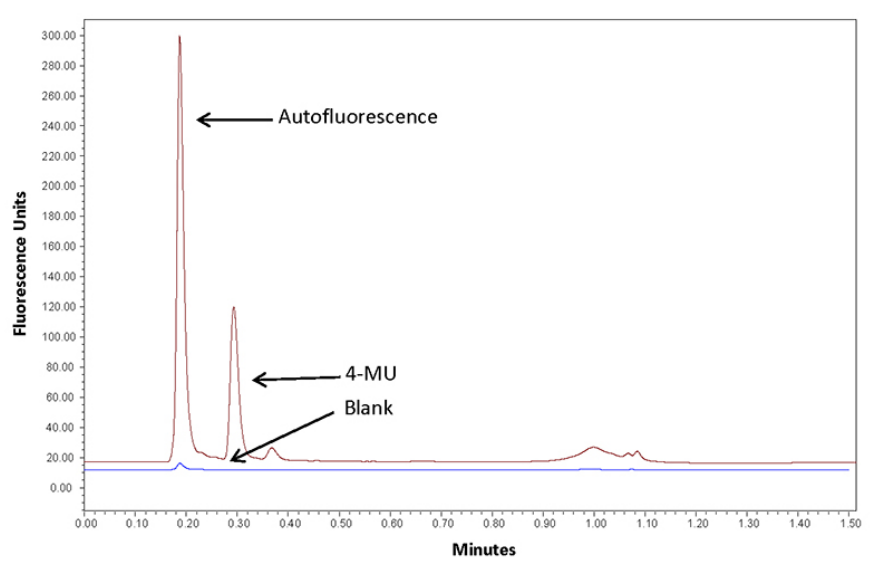

Figure 1. Separation of 4-MU from the endogenous, autofluorescent signal by UPLC. A UPLC system was developed that could reliably separate the endogenous, autofluorescent signal present in most biological samples from 4-MU. In this example a mouse spleen homogenate was used as the source of autofluorescence. Note the clear separation of the 4-MU and autofluorescent peaks, allowing for accurate and independent quantitation. Also note that this separation could be achieved in less than $1 \mathrm{~min}$.

the hydrolysis reactions have been carried out in individual steps. Also, to achieve enough 4-MU product for detection, a high substrate concentration and long incubation period (up to 2 days) are required. In our new method, a 1-step assay procedure was developed that could be performed in $1 \mathrm{hr}$. This method offers considerable advantages over the standard 4-MU assay currently available (Table 1).

At first, the optimal $\mathrm{pH}$ for both SGSH and $\alpha \mathrm{GD}$ was determined from 5 to 7 , revealing that a $\mathrm{pH}$ of 6.5 could detect both activities simultaneously. Next, three different $\mathrm{pH} 6.5$ buffers (sodium citrate, HEPES and Tris- $\mathrm{HCl}$ ) were compared, and HEPES buffer showed the highest 4-MU signal. In addition, the effects of ions and detergents on the SGSH/ $\alpha$ GD reaction were investigated. All ions were tested in the 1 to $100 \mathrm{mM}$ range, and detergents were tested in the $0.05 \%$ to $0.2 \%$ range. The data revealed that calcium had a significant positive impact at the $100 \mathrm{mM}$ concentration, and copper a dramatic negative effect at all concentrations (Fig. 2). Lithium and magnesium had intermediate positive effects, while potassium, sodium and manganese had little or no impact on the 4-MU signal. Three detergents (Igpal, Triton X-100, and Tween 20) also had no effect

Table 1. Comparison of the traditional vs. new $4 M U$ assay methods.

\begin{tabular}{lcc}
\hline Parameter & Traditional & New \\
\hline Final reaction volume $(\mu \mathrm{l})$ & 250 \\
Sample volume $(\mu \mathrm{l})$ & 20 & 50 \\
Substrate concentration $(\mathrm{mM}) 10$ & 10 & $>30$ \\
Reaction incubation time $(\mathrm{hr})$ & $\mathrm{nM}$ & $\mathrm{PM}$ \\
Sensitivity level & No & Yes \\
Separation of autofluorescence from 4-MU signal &
\end{tabular}




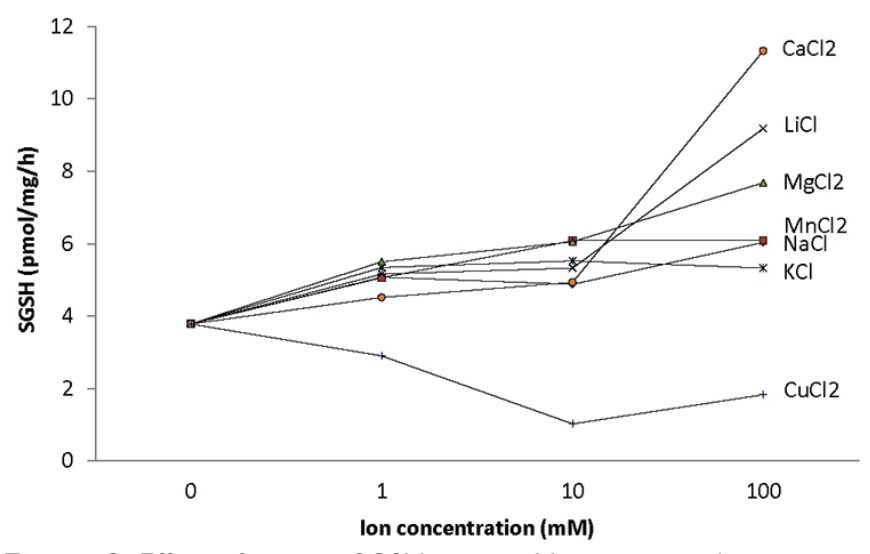

Figure 2. Effect of ions on SGSH activity. Using our newly developed 1-step UPLC assay method, we assessed the effect of various ions on $\mathrm{SGSH}$ activity. Note that $\mathrm{CaCl}_{2}$ had a highly positive effect on the activity, while $\mathrm{CuCl}_{2}$ was highly negative. For this reason our final reaction buffer contained $200 \mathrm{mM} \mathrm{CaCl}_{2}$.

on the SGSH/ $\alpha$ GD reaction (data not shown). Based on these results, for the standardized assay a $100 \mathrm{mM}$ HEPES buffer, $\mathrm{pH}$ 6.5 was used containing $200 \mathrm{mM} \mathrm{CaCl}_{2}$.

\section{Sensitivity of the One-Step Reaction}

To ensure detectable 4-MU levels in the 1-step reaction, the standard reaction was incubated at $37^{\circ} \mathrm{C}$ from 0.5 to $20 \mathrm{hr}$. When normalized by hour, the 4-MU levels were stable for up to $6 \mathrm{hr}$ of incubation. Importantly, after a $1 \mathrm{hr}$ incubation a reproducible 4-MU peak was detected in the UPLC system.
The limit of detection (LOD), defined as a signal-to-noise ratio of $3(\mathrm{~S} / \mathrm{N}=3)$, and the limit of quantification (LOQ) $(\mathrm{S} / \mathrm{N}=10)$ were determined for 4-MU under the optimized UPLC system conditions. The LOD for the 4-MU standard was $4.6 \mathrm{nM}$, and the LOQ was $13.5 \mathrm{nM}$. In contrast, the LOD using our fluorescent plate reader (BioTek, Synergy HT) was $198.3 \mathrm{nM}$ and the LOQ was $600.7 \mathrm{nM}$. Moreover, when the 4 -MU substrate was added to a mouse liver extract, the sensitivity of the reaction using the plate reader was further reduced due to quenching of the 4-MU signal from the endogenous, autofluorescent material (LOD and LOQ of $814.3 \mathrm{nM}$ and $2467.6 \mathrm{nM}$, respectively). In contrast, the LOD and LOQ were unchanged using the UPLC system.

The stability of the 4-MU product in the final reaction mixture ( $\mathrm{pH} 10.8$ to mimic the final reaction after stopping with sodium carbonate buffer) also was monitored over 5 days. There were no significant changes during the observation period. The Coefficient of Variation (CV) of repeat readings was $1.4 \%$.

\section{SGSH Activities in MPS IIIA Mouse Brains and Livers}

To verify the optimal conditions of our SGSH assay, mouse brain and liver homogenates from MPS IIIA, wild type and MPS IIIA heterozygous mice were studied. As shown in Fig. 3, after $1 \mathrm{hr}$ incubation the SGSH activities were significantly higher in wild type vs. MPS IIIA livers and brains. The residual SGSH activity in the MPS IIIA mice was $\sim 0.1 \%$ of wild type, confirming that the 4-MU product detected in the reaction was due to SGSH hydrolysis. Heterozygous MPS IIIA mice had intermediate activities that were also significantly different from wild type and homozygous MPS IIIA mice.

\section{Brain}

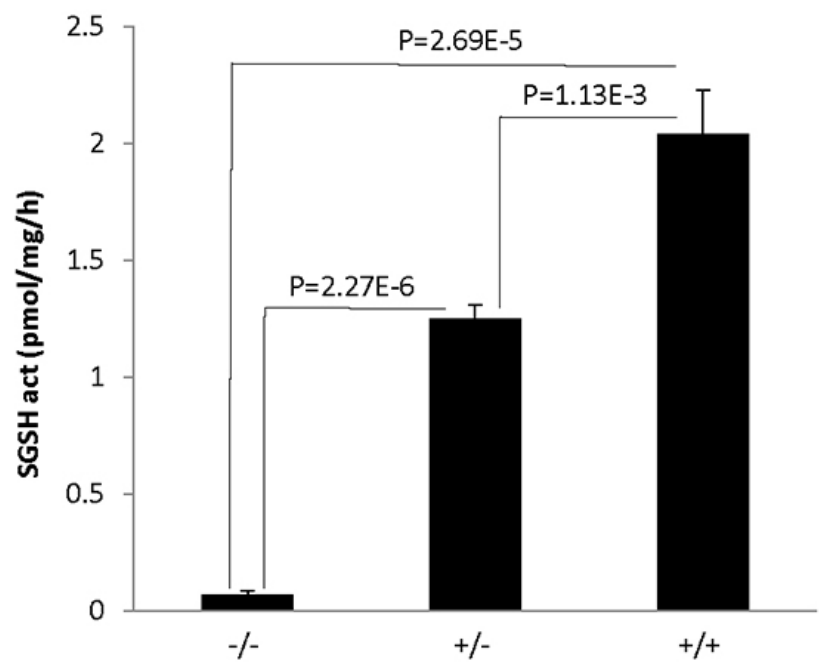

Liver

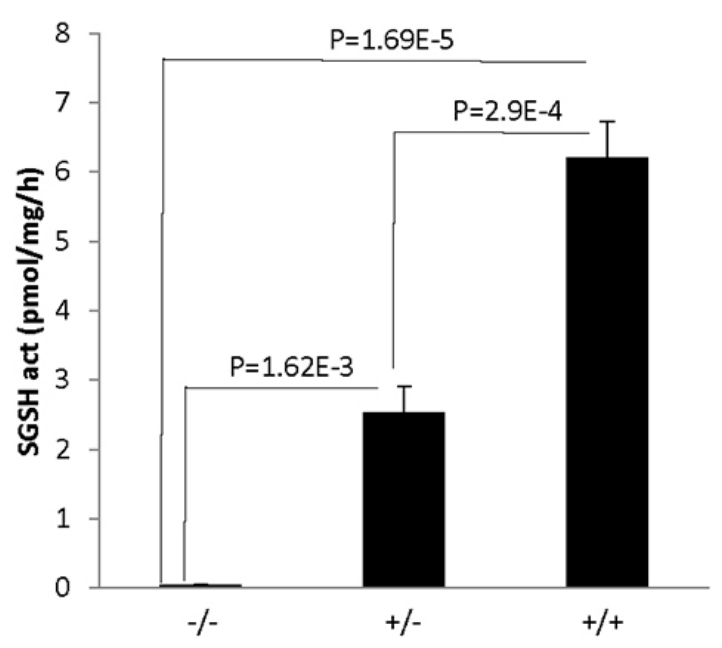

Figure 3. Validation of the UPLC assay using mouse tissue extracts. Brain and liver extracts were prepared from mice of three genotypes: MPS IIIA (-/-), heterozygous MPS IIIA (+/-) and wild type (+/+). For each genotype 8 mice were used. Note that the SGSH activity in MPS IIIA mice could be readily distinguished from heterozygous and wild type mice, and that the $\mathrm{p}$-values were highly significant. The activity in wild type mice also could be significantly distinguished from heterozygous mice. 
A.

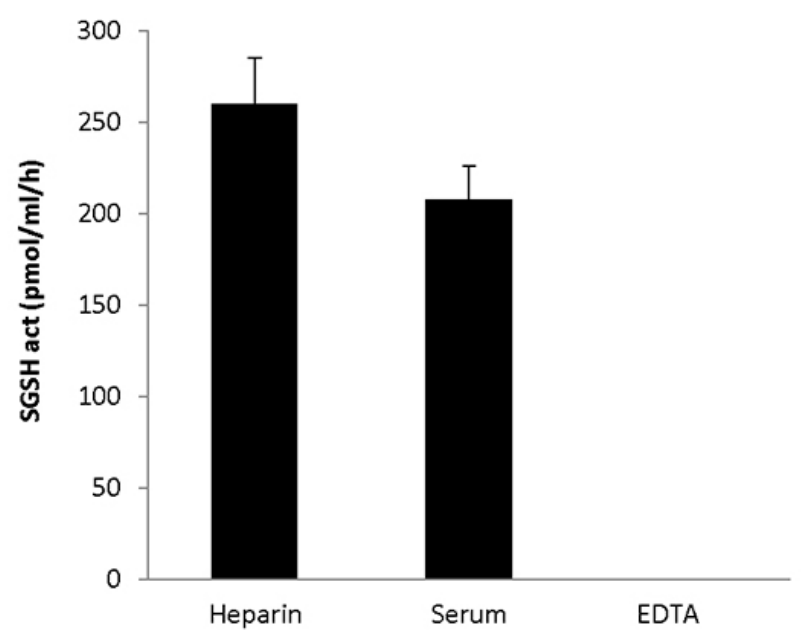

B.

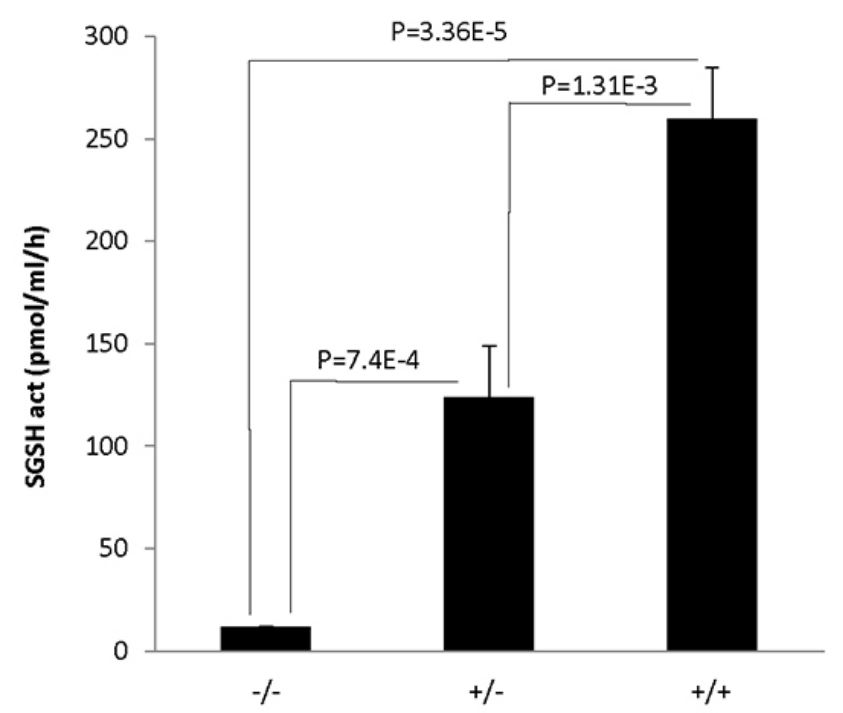

Figure 4. Validation of the UPLC assay using mouse plasma. (A) We first evaluated the effect of two common anti-coagulants on SGSH activity using our assay system. Note that there was no significant effect of collecting blood in the presence of heparin compared to collection without anti-coagulants (serum). However, collection of blood in EDTA had a highly inhibitory effect. We therefore used heparin (green top) tubes for all subsequent experiments. (B) To validate our new UPLC method using mouse plasma, blood was collected from the three genotypes: MPS IIIA (-/-), heterozygous MPS IIIA (+/-) and wild type (+/+). For each genotype 8 mice were used. Note that SGSH activity in the MPS IIIA mice could be readily distinguished from heterozygous and wild type mice, and that the p-values were highly significant. The activity in wild type mice also could be significantly distinguished from heterozygous mice.

\section{SGSH Activities in MPS IIIA Mouse Blood}

In general, very low lysosomal enzyme activities are present in plasma, either precluding accurate quantitation or requiring long incubation times. To determine whether our new UPLC assay method could detect SGSH activity in plasma, we first evaluated the effect of common anti-coagulants (heparin and EDTA) on the activity in mouse plasma, and found that EDTA was a strong inhibitor of the activity, while heparin had no significant impact (Fig. 4A). We therefore used heparin (green top) tubes to collect blood for subsequent experiments. Using this method the activity in mouse plasma was easily detected, even using a very small amount of blood $(5 \mu \mathrm{l})$ and a $1 \mathrm{hr}$ incubation time. The SGSH activities in plasma from the three MPS IIIA mouse genotypes were then measured (Fig. 4B), and highly significant differences were observed between homozygous MPS IIIA, heterozygous MPS IIIA and wild type mice.

\section{SGSH Activities in MPS IIIA Mouse DBS}

DBS have been widely used for disease screening [14]. To date, however, only one SGSH assay has been developed for DBS, and it requires the use of mass spectrometry equipment [10]. In Fig. 5 we show that our new assay method also can be used with mouse DBS. The highest SGSH activity was detected in wild

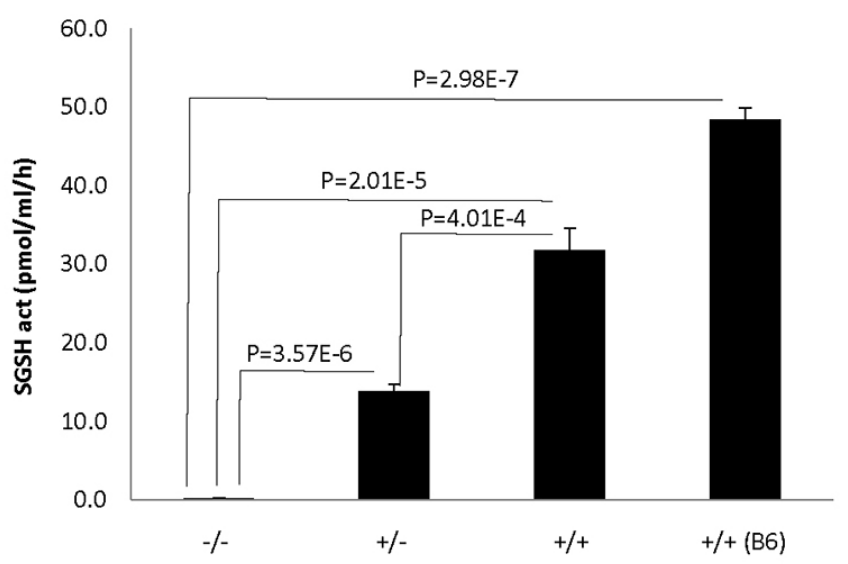

Figure. 5. Validation of the UPLC assay using mouse DBS. To validate our new UPLC method using mouse DBS, blood $(20 \mu \mathrm{l})$ was collected from the three genotypes: MPS IIIA (-/-), heterozygous MPS IIIA (+/-) and wild type (+/+) and applied to DBS cards according to the methods described in the text. For these experiments we also prepared DBS from pure B6 wild type mice for comparison. For each genotype 8 mice were used to prepare separate DBS cards. Note that the SGSH activity in MPS IIIA mice could be readily distinguished from heterozygous and wild type mice, and that the $\mathrm{P}$-values were highly significant. The activity in wild type mice also could be significantly distinguished from heterozygous mice. 
type DBS, and was highly correlated with the genotypes from the MPS IIIA mouse colony. Note that for these experiments we used wild type mice on two genetic backgrounds, pure B6 and a mixed background derived from the MPS IIIA colony. Compared to plasma (Fig. 4), the DBS activity was considerably lower, but also readily detected in wild type and heterozygous mice. The residual SGSH activity in homozygous MPS IIIA DBS was less than $0.5 \%$ that of wild type, further validating the specificity of this assay. Also, as with the plasma and tissue extracts, only $1 \mathrm{hr}$ incubation was required for the DBS assay, and heterozygous mice had activities that were intermediate between the homozygous and wild type animals.

\section{SGSH Activities in MPS IIIA Human Skin Fibroblasts and DBS}

To verify that our findings were not unique to mice, we next determined the SGSH activities in lysates from three human skin fibroblast cell lines (one homozygous MPS IIIA patient and two heterozygous individuals). As shown in Fig. 6, within $1 \mathrm{hr}$ our new assay method readily detected activity in the heterozygous cells, while the homozygous cells had virtually no detectable activity. To validate our method using human DBS, a DBS card was received containing de-identified blood from an MPS IIIA patient and parent. As shown in Fig. 7, our new method could readily distinguish the activities between the patient and heterozygous parent following $1 \mathrm{hr}$ incubation. Note that the SGSH activity detected in the human DBS was significantly lower than that in the mouse DBS (Fig. 5), but the differences between the MPS IIIA patient and parent were still highly significant.

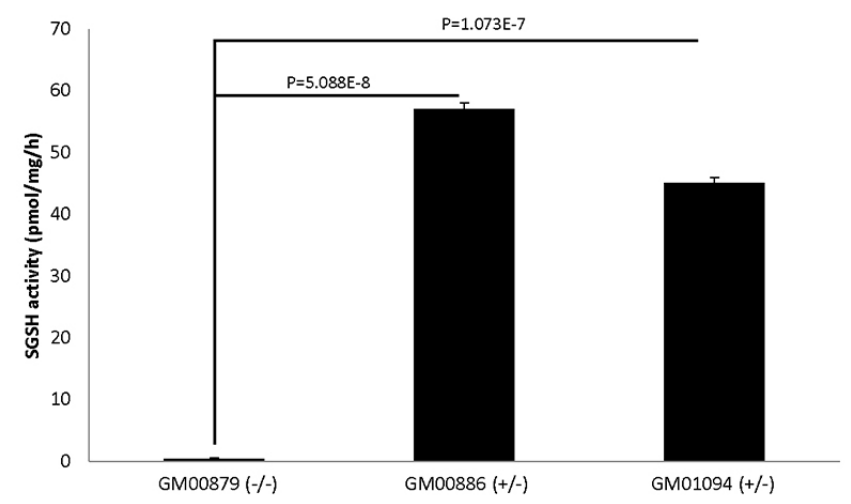

Figure 6. Validation of the UPLC assay using human skin fibroblasts. To determine whether our new assay method also could be used to detect human SGSH activity, we obtained cell lines from a patient with MPS IIIA (-/-) and two heterozygotes (+/-).

Note that the MPS IIIA patient could be readily distinguished from the two heterozygous individuals and that differences were highly significant. For this experiment all assays were performed in triplicate.

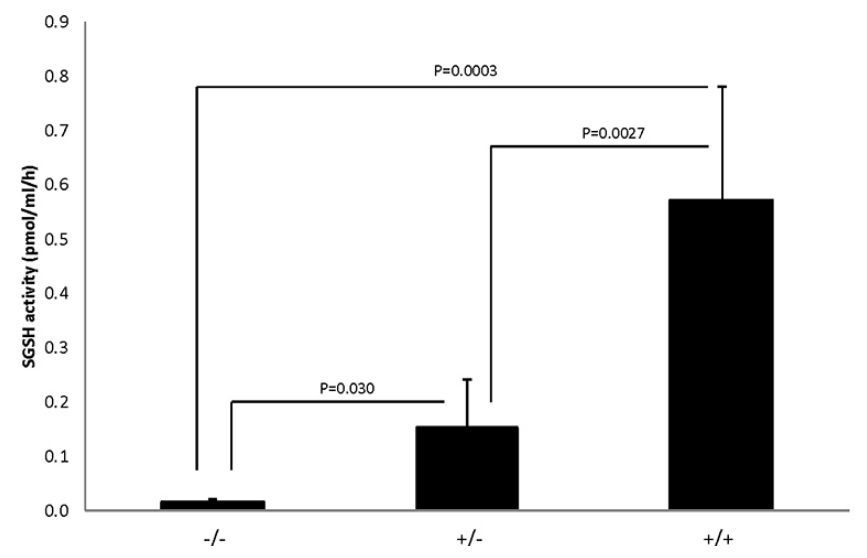

Figure 7. Validation of the UPLC assay using human DBS. To determine whether our new assay method also could be used to detect SGSH activity on human DBS, we obtained a DBS card containing blood from a patient with MPS IIIA (-/-) and heterozygous parent (+/-). For comparison, DBS cards were prepared from four healthy individuals $(+/+)$. Note that although the activity in the human DBS was $\sim 10$-fold lower than that found in mice (compare the $y$-axis in this figure to Fig. 5), the SGSH activity in the MPS IIIA patient could be readily distinguished from the parent and healthy individuals. For this experiment all assays were performed in triplicate.

\section{Discussion}

MPS IIIA is a devastating and fatal lysosomal storage disorder for which no treatment currently exists. Progressive neurologic disease dominates the clinical picture, although other organ systems, including the skeleton and liver, also are involved. In recent years there has been considerable research around the development of disease modifying therapies for this disorder, and several clinical trials examining CNS-directed gene or enzyme replacement therapies are currently underway $[4,5]$. This has led to considerable research surrounding SGSH, highlighting the need for inexpensive, easy and reliable activity assays.

In 1996 Karpova et al. [7] developed a fluorogenic, 4-MU based assay for the detection of SGSH activity, and demonstrated its ability to diagnosis MPS IIIA patients. However, this assay method required two independent enzyme steps, the first using the SGSH enzyme source (e.g., cell or tissue extracts) to de-sulfate the $4 \mathrm{MU}-\mathrm{GlcNS}$, and the second using yeast GD to release the 4-MU. The resulting fluorescence was then quantified using a fluorometer or 96-well plate reader. Due to the use of two enzymes in independent reactions, to reliable quantify SGSH activity long incubation times were required and the assay procedure required two days. Even so, the low sensitivity of this procedure precluded its adaptation to DBS. More recent assays have used mass spectrometry, and are significantly more sensitive, allowing them to be adapted for DBS [8-10]. However, research laboratories may not have routine access to LC-MS/ 
MS equipment, and to detect SGSH activity on DBS overnight (16 hr) incubations are still required.

We therefore sought to develop an alternative assay that could be used for routine research purposes. We focused on the $4 \mathrm{MU}$-GlcNS substrate since it is commercially available and inexpensive. We first established UPLC conditions to separate the 4-MU signal from the endogenous, autofluorescent material present in biological samples since this material can often interfere with accurate quantitation of the 4-MU signal [15]. In our method these signals could be separated in under 1 min, allowing accurate and independent quantitation.

Next, we examined various buffer conditions so that we could determine SGSH and $\alpha \mathrm{GD}$ in the same reaction mixture. We settled on a simple HEPES buffer at a $\mathrm{pH}$ of 6.5 , and included $200 \mathrm{mM}$ calcium chloride since calcium cations significantly enhanced the combined SGSH/ $\alpha$ GD reaction. To our knowledge this is the first demonstration of the effect of calcium cations on SGSH activity, as well as the inhibitory effect of EDTA on this reaction.

To verify this assay method, we first used biological samples from the MPS IIIA mice. In brain and liver homogenates, as well as plasma, the residual SGSH activity in homozygous MPS IIIA mice using this new assay was less than $0.5 \%$ of wild type. Of interest, heterozygous MPS IIIA mice had intermediate activity values, suggesting that this assay may be suitable for carrier detection, although more samples must be studied and the method validated for this purpose. These results were further validated using MPS IIIA patient and heterozygous skin fibroblasts.

The enhanced sensitivity of our UPLC system $(>50$-fold compared to a fluorescent plate reader) also permitted us to adapt this method to DBS, and we found that we could reliably identify MPS IIIA affected mice or patients after only $1 \mathrm{hr}$ incubation. The values using human DBS were much lower than that from mice, but none-the-less the SGSH activity was readily detected in healthy individuals and an MPS IIIA parent (obligate heterozygote), and significantly less in the MPS IIIA patient. As noted above, the only available DBS assay for SGSH uses mass spectrometry and requires an overnight incubation. It should be noted that the sensitivity of our assay relies in part on the UPLC equipment available, and specifically the type of fluorescence detector. In our laboratory we used a model B10UPF823M UPLC fluorescence detector (Waters) and compared to a BioTek, Synergy HT fluorescent plate reader.

Thus, this new assay procedure offers high sensitivity and a single vs. two step procedure, allowing for a short (1 $\mathrm{hr}$ ) incubation period, even with human DBS. It also uses the commercially available and inexpensive $4 \mathrm{MU}-\mathrm{GlcNS}$ substrate. The assay has been validated using mouse samples and can reliably identify MPS IIIA vs. normal or heterozygous animals. We also provide preliminary data with human samples indicating that the same methodology can be used. Future studies with additional human samples will further validate the findings. We believe that this method is an alterative that could be readily adapted by many research laboratories studying SGSH, and perhaps be useful for diagnostic and screening purposes as well.

\section{Acknowledgements}

The authors wish to acknowledge the expert technical assistance of Mr. Victor DeAngelis and Ms. Changzhi Zhu for assistance with this work.

\section{Declaration of Conflicting Interests}

The authors declare no conflicting interests for this work.

\section{References}

1. Wagner VF, Northrup H. Mucopolysaccharidosis type III. In: Adam MP, Ardinger HH, Pagon RA, et al. ed. GeneReviews ${ }^{\circ}$. University of Washington, Seattle; 19932020: Updated September 19, 2019. https://www.ncbi.nlm. nih.gov/books/NBK546574/

2. Sorrentino NC, Cacace V, De Risi M, et al. Enhancing the therapeutic potential of sulfamidase for the treatment of mucopolysaccharidosis IIIA. Mol Ther Methods Clin Dev. 2019;15:333-342. doi: 10.1016/j.omtm.2019.10.009

3. Bigger BW, Begley DJ, Virgintino D, Pshezhetsk AV. Anatomical changes and pathophysiology of the brain in mucopolysaccharidosis disorders. Mol Genet Metab. 2018;125:322-331. doi: 10.1016/j.ymgme.2018.08.003

4. Marcó S, Haurigot $\mathrm{V}$, Bosch F. In vivo gene therapy for mucopolysaccharidosis type III (Sanfilippo Syndrome): A new treatment horizon. Hum Gene Ther. 2019;30:1211-1221. doi: 10.1089/hum.2019.217

5. Boado RJ, Lu JZ, Hui EK, Pardridge WM. Reduction in brain heparan sulfate with systemic administration of an IgG trojan horse-sulfamidase fusion protein in the mucopolysaccharidosis type IIIA mouse. Mol Pharm. 2018;15:602-608. doi: 10.1021/acs.molpharmaceut.7b00958

6. Civallero G, De Mari J, Viapiana Camelier M, Burin M, Giugliani R. Assay of heparan-N-sulfamidase in dried leukocytes impregnated in filter paper: A new tool for the identification of Mucopolisaccharidosis IIIA and potentially other lysosomal disorders. Mol Gen Metab. 2013;108(4):267-268. doi: 10.1016/j.ymgme.2013.02.001.

7. Karpova EA, Voznyi YaV, Keulemans JL, et al. A fluorimetric enzyme assay for the diagnosis of Sanfilippo disease type A (MPS IIIA). J Inherit Metab Dis. 1996;19:278-285. doi: 10.1007/BF01799255 
8. Wolfe BJ, Ghomashchi F, Kim T, Abam CA, Sadilek M, Jack R, Thompson JN, Scott CR, Gelb MH, Turecek F. New substrates and enzyme assays for the detection of mucopolysaccharidosis III (Sanfilippo Syndrome) types A, B, C, and D by tandem mass spectrometry. Bioconjug Chem. 2012;23:557-564. doi: 10.1021/bc200609x

9. Spacil Z, Tatipaka H, Barcenas M, Scott CR, Turecek F, Gelb MH. High-throughput assay of 9 lysosomal enzymes for newborn screening. Clin Chem. 2013;59:502-511. doi: 10.1373/clinchem.2012.189936

10. Yi F, Hong X, Kumar AB, et al. Detection of mucopolysaccharidosis III-A (Sanfilippo Syndrome-A) in dried blood spots (DBS) by tandem mass spectrometry. Mol Genet Metab. 2018;125:59-63. doi: 10.1016/j. ymgme.2018.05.005

11. Bhaumik M, Muller VJ, Rozaklis T, et al. A mouse model for mucopolysaccharidosis type III A (Sanfilippo syndrome). Glycobiology. 1999;9:1389-1396. doi: 10.1093/ glycob/9.12.1389
12. Gliddon BL, Hopwood JJ. Enzyme-replacement therapy from birth delays the development of behavior and learning problems in mucopolysaccharidosis type IIIA mice. Pediatr Res. 2004;56:65-72. doi: 10.1203/01. PDR.0000129661.40499.12

13. Blanch L, Weber B, Guo XH, Scott HS, Hopwood JJ. Molecular defects in Sanfilippo Syndrome type A. Human Molecular Genetics. 1997;5:787-791. doi: 10.1093/ $\mathrm{hmg} / 6.5 .787$

14. Ombrone D, Giocaliere E, Forni G, Malvagia S, la Marca G. Expanded newborn screening by mass spectrometry: New tests, future perspectives. Mass Spectrum Rev. 2016;35:7184. doi: $10.1002 /$ mas. 2146

15. Kongkamnerd J, Milani A, Cattoli G, et al. The quenching effect of flavonoids on 4-methylumbelliferone, a potential pitfall in fluorimetric neuraminidase inhibition assays. J Biomol Screening. 2011;16(7):755-782. doi: $10.1177 / 1087057111409221$ 